NOUVELL

\section{Migration collective : un partage des tâches entre cellules leaders et coordination supracellulaire}

Myriam Reffay ${ }^{1,2}$, Maria-Carla Parrini ${ }^{3}$, Olivier Cochet-Escartin ${ }^{1}$, Benoit Ladoux ${ }^{2,4}$, Axel Buguin ${ }^{1}$, Sylvie Coscoy ${ }^{1}$, François Amblard ${ }^{1}$, Jacques Camonis ${ }^{3}$, Pascal Silberzan ${ }^{1}$

$>$ La migration cellulaire collective, au cours de laquelle les cellules restent liées les unes aux autres tout en se déplaçant, est un processus fondamental qui intervient dans la morphogenèse, la réponse immunitaire, la régénération tissulaire mais aussi le cancer [1]. La migration collective conduit généralement à une hiérarchie entre cellules, liée à la position de ces dernières dans l'assemblage. Ainsi, des structures organisées apparaissent souvent sous la forme de digitations, avec à leur tête des cellules dites leader suivies par un groupement de cellules nommé doigt de migration qui entraîne ensuite le reste du tissu [2, 3]. Ces structures sont notamment observées lors de la migration collective de cellules épithéliales induite par simple libération d'espace libre au moyen de micro-pochoirs [3] ou par blessures [2], mais aussi in vivo lors de l'invasion tumorale [1] ou la morphogenèse [4]. La migration collective s'appuie sur les principes mécanistiques de la migration individuelle, et notamment sur la contractilité du cytosquelette acto-myosine; toutefois elle met aussi en jeu les adhésions intercellulaires, la communication entre cellules, et implique une coordination de l'activité du cytosquelette $(\rightarrow)$ [12]. Dans ce contexte, le profil de force généré par un doigt est une informa-

$\rightarrow$ Voir la Synthèse de Éric Theveneau et Nicolas David, page 751 de ce numéro tion essentielle pour déterminer le rôle mécanique de la cellule leader, car l'organisation mécanique de la migration collective est encore peu étudiée. En outre, les processus de coordination impliquent une distribution d'activité biochimique à l'intérieur des cellules. On pense en particulier aux RhoGTPases comme RhoA et Racl, essentielles à la migration de cellules individuelles [5], mais dont l'action à l'échelle collective n'a été explorée que très récemment $[6,7]$. Afin de répondre à ces questions essentielles, il est nécessaire de développer des outils adaptés à l'échelle collective.

Microscopie multiparamétrique :

forces mécaniques et activité

protéique

Dans l'étude que nous avons récemment publiée dans Nature Cell Biology [8], la migration collective est obtenue grâce

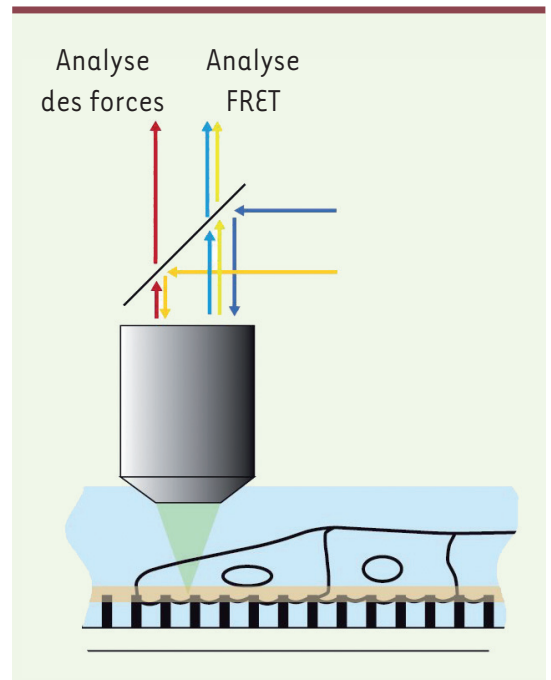

${ }^{1}$ Laboratoire physico-chimie Curie, UMR 168, Institut Curie, centre de recherche, CNRS, UPMC, Paris, F-75248, France ;

${ }^{2}$ Laboratoire matière et systèmes complexes - UMR 7057, Université Paris Diderot, CNRS, Paris, F-75251, France ;

${ }^{3}$ groupe analyse des réseaux de transduction, U830, Institut Curie, centre de recherche, Inserm, Paris, F-75248, France.

${ }^{4}$ Adresses actuelles : Institut Jacques Monod (IJM), CNRS UMR 7592 et Université

Paris Diderot, Paris F75205, France ;

Mechanobiology Institute, National University of Singapore 117411, Singapore.

myriam.reffay@univ-paris-diderot.fr

à des blessures modèles; le moteur de cette migration est donc la simple libération d'espace libre. Nous avons corrélé deux types essentiels d'informations (Figure 1) : les forces développées par les cellules sur le substrat et l'activité des RhoGTPases dans le plan basal des cellules. Les cellules sont déposées sur un tapis de micropiliers dont la déflexion renseigne sur les forces mises en jeu (amplitude et direction), ce qui permet d'obtenir une carte dynamique des forces [9]. Des biosenseurs moléculaires [10], dont la détection est basée sur une technique FRET (fluorescence resonance energy transfer), sont utilisés afin de déterminer l'activité des RhoGTPases RhoA et Racl. Ces sondes renseignent sur la conformation des GTPases via l'interaction intramoléculaire de deux fluorophores.

Figure 1. Dispositif d'imagerie multiparamétrique : coenregistrement des forces et de l'activité de la protéine RhoA. Les forces sont mesurées par la déflexion des piliers souples sur lesquels les cellules migrent [9]. La position du sommet de ces piliers (proportionnelle à la force qui y est appliquée) est mesurée en fluorescence sur le canal rouge (Cy5). En parallèle, l'activité de RhoA est mesurée via le signal FRET dû au changement de conformation de la protéine lorsqu'elle devient active (ratio YFP/CFP). L'ensemble des mesures sont effectuées en microscopie confocale dans le plan basal. D'après [8]. 
Au moyen d'un microscope confocal, on suit simultanément la génération de forces et la distribution d'activité GTPase dans les doigts de migration au niveau du plan basal des cellules. Ces mesures peuvent être combinées avec différentes techniques de perturbation de l'assemblage cellulaire, comme l'utilisation de drogues ou l'interférence ARN, afin d'inhiber l'activité des GTPases, mais aussi la microdissection laser qui permet de couper localement les fibres d'actine [11].

\section{Mécanique des doigts de migration :} identification d'une super-cellule La cartographie des forces à l'échelle du doigt de migration a mis en évidence le rôle prédominant de la cellule leader. Tout d'abord, focalisons nous sur les forces générées suivant l'axe longitudinal ou axe de progression du doigt. La cellule leader exerce une traction très importante qui permet de tirer les cellules qui lui font suite. Les forces de traction développées le long du doigt dépendent de la position relative des cellules dans l'assemblage. Ainsi, à mesure que l'on s'éloigne de la cellule leader, les forces de traction diminuent pour s'annuler et, dans la dernière partie du doigt, les cellules sont tirées (forces négatives) (Figure 2). On reproduit à l'échelle pluricellulaire la cartographie des forces obtenue pour une cellule unique, avec un front qui tracte l'ensemble.

Si on s'intéresse ensuite à la composante perpendiculaire, on repère une zone contractile sur les bords du doigt corrélée avec la présence d'un câble pluricellulaire d'acto-myosine. Des expériences de microdissection ont montré que ce câble est responsable à la fois de l'intégrité du doigt de migration - en prévenant la naissance de cellules leader multiples -, mais intervient aussi dans la transmission des forces le long du doigt.
En effet, ce câble est sous tension car son ablation en un point conduit à une rétraction rapide et à l'annulation des forces à l'échelle de plusieurs cellules en amont ou en aval de la dissection. De plus, la rupture de ce câble conduit à la naissance de doigts de migration annexes.

L'équilibre mécanique est réalisé à l'échelle du doigt, et celui-ci constitue donc une entité mécanique cohérente qui reproduit à l'échelle pluricellulaire le diagramme mécanique d'une cellule unique : l'avant du doigt opère la traction et l'arrière est tiré. On peut qualifier ces doigts de «super-cellules ».

\section{Action de la protéine RhoA :} proportionnalité entre gradient

\section{d'activité et forces}

Les RhoGTPases ont un rôle essentiel dans la fonctionnalité du cytosquelette d'actine à l'échelle de la cellule individuelle. La protéine RhoA possède un

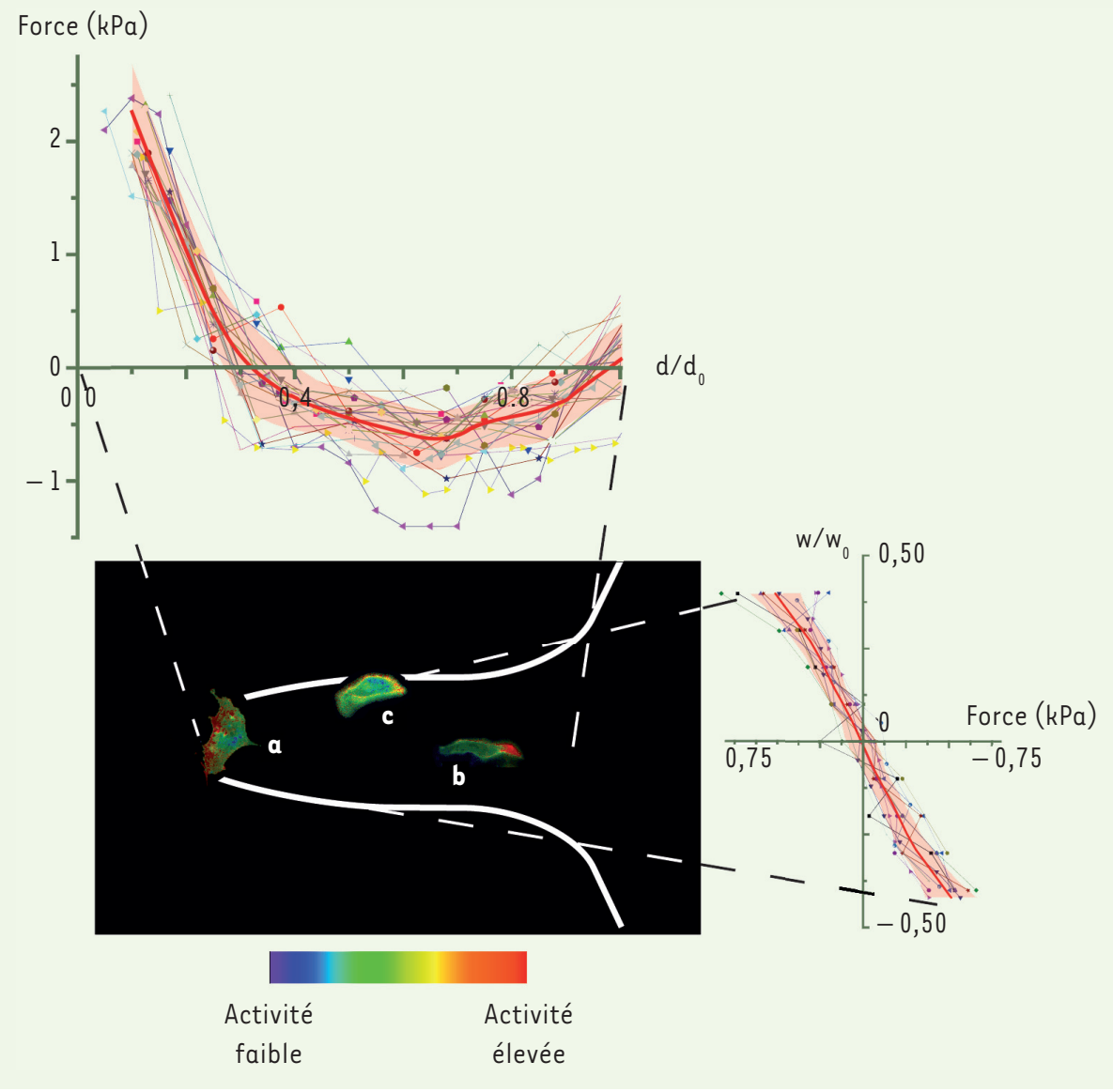

Figure 2. Forces et activités différentielles dans un doigt de migration. L'image centrale est un montage représentant un doigt de migration typique. Les couleurs traduisent l'activité de RhoA. Dans la cellule leader, l'activité de la protéine RhoA est maximale à l'avant (cellule a). Au contraire, pour les cellules du doigt, on note la distribution inverse (cellule b). De plus, l'activité de RhoA est importante sur les bords (cellule $\mathrm{c}$ ). Sont également représentés les profils de forces dans les deux directions. Dans la direction longitudinale (panneau du haut), on observe une force de traction très importante au niveau du leader qui s'inverse dans le doigt. Dans la direction transversale (panneau de droite), les forces sont centripètes à partir des bords du doigt (d'après [8]). 
double rôle : à l'avant de la cellule, elle stabilise les adhésions focales afin de permettre aux fibres de stress d'adhérer fortement et de tracter la structure, à l'arrière elle génère une contraction actine-myosine afin de rétracter l'arrière de la cellule. Il est donc particulièrement intéressant d'établir une cartographie de l'activité de cette protéine à l'échelle des doigts de migration.

Dans les mouvements collectifs, la protéine RhoA a toujours les mêmes fonctions, mais celles-ci sont distribuées sur l'ensemble du doigt de migration. $\varepsilon$ n utilisant des drogues inhibitrices ou des siARN ciblés contre RhoA, nous avons établi que cette protéine contrôle la formation des doigts de migration. Toutefois, plus que l'activité elle-même, c'est l'activité différentielle, qui correspond au gradient entre l'avant et l'arrière des cellules (gradient longitudinal) ou entre ses deux côtés latéraux (gradient transversal), qui est le paramètre à suivre en fonction de la position des cellules dans le doigt.

Ces activités différentielles reproduisent les mêmes variations que les forces de traction (décrites ci-dessus) dans les directions longitudinale et transversale (Figure 2). Un coefficient de proportionnalité de $7 \pm 2 \mathrm{kPa}$ est obtenu entre les forces et les activités différentielles. Il décrit la sensibilité de la réponse mécanique au gradient d'activité de la protéine RhoA. Deux types de mesures viennent confirmer ce phénomène: tout d'abord, des mesures à l'échelle de la cellule individuelle, qui comparent directement les forces générées par cette dernière à l'activité différentielle de
RhoA à l'intérieur de celle-ci, mais aussi des mesures effectuées sur des cellules appartenant à plusieurs doigts en renormalisant leurs positions par la longueur et la largeur du doigt. On décrit ainsi sur un doigt «moyen » à la fois le gradient de la protéine RhoA et les forces.

L'utilisation d'inhibiteurs a aussi permis de mettre en évidence deux types de migration collective : le premier, lorsque RhoA est actif, se caractérise par le rôle prépondérant de la cellule leader dans la traction de l'assemblage, mais aussi l'action de traction des groupes cellulaires les plus proches; le second, lorsque RhoA est désactivé, donne de l'importance aux cellules de l'épithélium qui exercent une poussée du front de migration. Deux modes de propulsion du front de migration, par traction ou par poussée du front, sont ainsi mis en évidence et dépendent du gradient local d'activité de RhoA.

En conclusion, l'utilisation d'une microscopie multiparamétrique a permis de mettre en évidence des processus essentiels nécessaires à la migration collective. Au sein de doigts de migration, les cellules coordonnent leur cytosquelette d'actine et utilisent une contractilité dirigée par l'activité différentielle de la protéine RhoA au niveau cellulaire. Elles joignent ainsi leurs forces et agissent de concert pour conserver une structure hiérarchisée. Au sein de ces «super-cellules», on retrouve à une échelle supracellulaire des processus similaires à ceux mis en œuvre lors de la migration de cellules individuelles. $\diamond$

Collective cell migration: a fair sharing between leader cells and supracellular coordination

\section{REMERCIEMENTS}

Nous remercions le C'nano île-de-France, l'Association Christelle Bouillot et l'Association pour la recherche sur le cancer pour leur soutien financier.

\section{LIENS D'INTÉRÊT}

Les auteurs déclarent n'avoir aucun lien d'intérêt concernant les données publiées dans cet article.

\section{RÉFÉRENCES}

1. Friedl P, Gilmour D. Collective cell migration in morphogenesis, regeneration and cancer. Nat Rev Mol Cell Biol $2009 ; 10: 445-57$.

2. Omelchenko T, Vasiliev JM, Gelfand IM. Rhodependant formation of epithelial leader cells during wound healing. Proc Natl Acad Sci USA 2003; 100 : 10788-93.

3. Poujade M, Grasland-Mongrain $\varepsilon$, Petitjean L, et al. Sociologie des cellules épithéliales. Med Sci (Paris) 2008 ; 24 : 684-6.

4. Wood W, Jacinto A, Grose R, et al. Wound healing recapitulates morphogenesis in drosophila embryos. Nat Cell Biol 2002 ; 4 : 907-12.

5. Primeau M, Lamarche-Vane N. Coup d'oeil sur les petites GTPases Rho. Med Sci (Paris) 2008 ; 24 : 157-62.

6. Theveneau $\varepsilon$, Marchant L, Kuriyama S, et al. Collective chemotaxis requires contact dependent cell polarity. Dev Cell 2010 ; 19 : 39-53.

7. Gagglioli C, Hooper S, Hidalgo-Carcedo C, et al. Fibroblast-led collective invasion of carcinoma cells with differing roles for RhoGTPases in leading and following cells. Nat Cell Biol 2007 ; 9 : $1392-400$.

8. Reffay M, Parrini MC, Cochet-Escartin 0 , et al. Interplay of RhoA and mechanical forces in collective cell migration driven by leader cells. Nat Cell Biol $2014 ; 16: 217-23$.

9. du Roure 0, Saez A, Buguin A, et al. Force mapping in epithelial cell migration. Proc Natl Acad Sci USA $2005 ; 102: 2390-5$.

10. Aoki K, Matsuda M. Visualization of small GTPase activity with fluorescence energy transfer-based biosensors. Nat Prot 2009 ; 4 : 1623-31.

11. Colombelli J, Pepperkok R, Stelzer EHK, Reynaud \&G. La nanochirurgie laser en biologie cellulaire. Med Sci (Paris) $2006 ; 22: 651-8$.

12. Theveneau $\varepsilon$, David N. Migrations cellulaires collectives. Med Sci (Paris) 2014 ; 30 : 751-7.

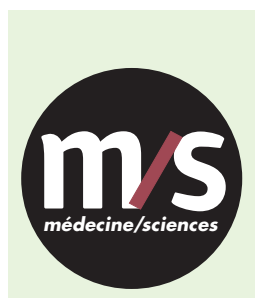

Tarifs d'abonnement $m / s-2014$

> Grâce à $m / s$, vivez en direct les progrès

Tarifs d'abonnement $m / s-2014$ des sciences biologiques et médicales

Abonnez-vous

à médecine/sciences

Bulletin d'abonnement page 813 dans ce numéro de $\mathrm{m} / \mathrm{s}$

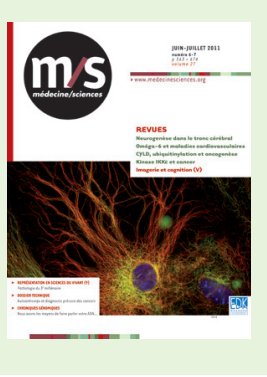

